\title{
Effects of changes in drinking habits on lifestyle-related diseases
}

\author{
Seiichiro KoJima $^{1}$ Norihito Watanabe ${ }^{1}$ Shinji Takashimizu $^{2}$ Kazuya AnzaI $^{1}$ Shingo Tsuda $^{1}$

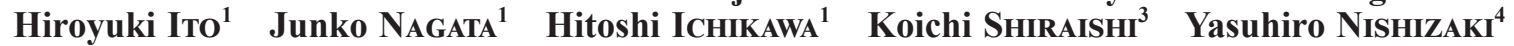 \\ ${ }^{I}$ Division of Gastroenterology, Tokai University Hachioji Hospital \\ ${ }^{2}$ Department of Clinical Health Science, Tokai University School of Medicine \\ ${ }^{3}$ Division of Gastroenterology, Tokai University Tokyo Hospital \\ ${ }^{4}$ Division of Clinical Health Science, Tokai University Tokyo Hospital
}

\begin{abstract}
OGjectives Drinking habits are closely associated with various lifestyle-related diseases. We investigated the effects of changes in alcohol consumption on lifestyle-related disease in subjects who underwent multiple health check-ups at the Tokai University Hospital Health Check-up Center.

Design Study of causation.

Methods The subjects were 19,382 repeated examinees who underwent multiple health check-ups at the Health Checkup Center in our hospital from 2005 to 2015. Using the questionnaire method, the drinking quantity was estimated, and the effect of the change on the parameter (body mass index (BMI), systolic blood pressure (SBP), low-density lipoprotein cholesterol (LDL-C), high-density lipoprotein cholesterol (HDL-C), triglycerides (TG), fasting blood glucose (FBG), uric acid (UA)) of the life-style related disease was analyzed, when the drinking quantity changed in first health check-ups
\end{abstract} and last health check-ups.

Resufts BMI, SBP, TG, FBG, and UA all worsened in a dose-dependent manner with increases in alcohol consumption, and improved when alcohol consumption decreased. HDL-C improved with increased alcohol consumption in a dosedependent manner, and worsened with a decrease in alcohol consumption. The increase in alcohol consumption contributed to the development of metabolic syndrome, but it was through the deterioration of the parameters of lifestyle-related diseases and was not an independent factor.

Conclusion Increased alcohol consumption during the course of observation positively affected HDL-C but negatively affected all other lifestyle-related disease parameters. (HEP. 2019; 46: 431-437.)

Key words Alcohol consumption, health check-up, lifestyle-related disease, fatty liver, metabolic syndrome

\section{Introduction}

Drinking habits are closely associated with various lifestylerelated diseases, including obesity, hypertension, diabetes, dyslipidemia, hyperuricemia, and fatty liver ${ }^{1}$. We previously surveyed alcohol consumption status and parameters for various lifestylerelated diseases in people examined at the Tokai University Hospital Health Check-up Center ${ }^{2}$. The results showed a large sex difference in those who consumed alcohol regularly, which was $75.2 \%$ in men and $37.1 \%$ in women. By age, the frequency of consumption was seen to increase with age in men, but to gradually decrease with age in women. The relationships between lifestyle-related disease parameters and alcohol consumption were investigated using multivariate logistic analysis. The results suggested that low alcohol consumption had positive effects on body mass index (BMI), low-density lipoprotein (LDL) cholesterol (LDL-C), high-density lipoprotein cholesterol (HDL-C), and triglycerides (TG). In contrast, high alcohol consumption was shown to negatively affect systolic blood pressure (SBP), TG,

\footnotetext{
Received: May 14, 2019, Accepted: June 10, 2019

Corresponding author: Seiichiro Kojima

'Address; Tokai University Hachioji Hospital

1838 Ishikawa-machi, Hachioji, Tokyo 192-0032, Japan

TEL: +81-42-639-1111, FAX: +81-42-639-1144

E-mail: jbc01232@nifty.com
}

fasting blood glucose (FBG), and uric acid (UA) ${ }^{2)}$.

Here, we report an investigation of the effects of changes in drinking habits on lifestyle-related diseases in people who underwent multiple health check-ups at the Tokai University Hospital Health Check-up Center.

\section{Materials and Methods}

Over the 11 years from 2005 to 2015, 169,326 health checkups were performed at our hospital's Health Check-up Center. After excluding 7,910 cases for which alcohol consumption data were missing, data from 161,416 check-ups were analyzed. The total number of people examined was 37,213. After excluding 12,532 people who were examined only once, there were 24,681 people who underwent multiple health check-ups. Next, to eliminate the effect of medical care and drug administration, we excluded check-ups when patients were receiving treatment for hypertension, dyslipidemia, diabetes, and hyperuricemia, which accounted for 28,231 check-ups (17.5\%), 11,781 check-ups (7.3\%), 8,548 check-ups (5.3\%) and 6,335 check-ups (3.9\%), respectively. Finally, the analysis was conducted with data from 117,730 health check-ups for 19,382 people.

The subjects were 10,757 men $(55.5 \%)$ and 8,625 women $(44.5 \%)$. At the time of evaluation, their ages ranged from 21 to 98 years old, with a mean age of $57.8 \pm 10.9$ years. The mean age of men was $58.2 \pm 11.0$ years and that of women was $57.4 \pm 10.8$ 
years. The median age distribution was 59 years, the 25 th percentile was 50 years and the 75 th percentile was 65 years. The mean follow-up period was $2,008 \pm 1,124$ days. For repeaters more than 3 times, data from the first and last check-up were used.

To survey alcohol consumption, a questionnaire was used that asked the type of alcohol most frequently consumed (multiple responses accepted), the amount consumed, and the frequency of consumption. Responses were selected from among "do not drink," "1 day a week," "2 to 5 days a week," and "6 or more days per week." The amount of pure ethanol was converted from the type of alcohol, and the approximate amount consumed per day was calculated by multiplying by a coefficient of $1 / 7,1 / 3$, or $1 / 1$ depending on the frequency of consumption. The parameters used to assess lifestyle-related disease were BMI, SBP, LDL-C, HDL-C, TG, FBG, and UA.

JMP $^{\circledR} 12$ (SAS Institute Inc., Cary, NC, USA) was used for the statistical analysis, and Tukey-Kremer's HSD multiple comparison, an analysis of variance and a logistic analysis were performed.

This study was performed in accordance with the epidemiological research protocol of our hospital. A preliminary review was conducted, health check-up examinees were informed about the study, and anonymity was maintained. The data used in this study are "Those anonymized so as can not to identify specific individuals".

\section{Results}

\section{Relationship between alcohol consumption and markers related to alcohol consumption}

The relationship between the amount of alcohol consumed and $\gamma$-GTP, a marker of alcohol consumption, is shown in Fig. 1 . Alcohol consumption were divided into five groups: "Do not drink," " $\leq 10$ g/day," " $\leq 20$ g/day," " $\leq 40$ g/day," and " $>40$ g/ day," and the value of $\gamma$-GTP was shown in a box plot. The distribution of $\gamma$-GTP was asymmetrical with a gentle deviation to the high side, and the median value of $\gamma$-GTP showed a higher value with an increase in the alcohol consumption. Tukey-Kremer's HSD multiple comparison showed significant differences among all groups $(\mathrm{p}<0.0001)$.

\section{Changes in drinking habits during the follow-up period}

The number of people who changed their alcohol consumption during the mean follow-up period of 2,008 days is shown in Table 1. To assess changes in alcohol consumption, they were divided into five groups as described above.

They were weighted 1, 2, 3, 4 each, and when a change in drinking habits was seen during the follow-up period, the size of the change was expressed with weighted differences. This is shown as $\Delta$ alcohol consumption. When $\Delta$ alcohol consumption is 0 , there is no change in drinking habit. A positive increase in $\Delta$ alcohol consumption indicates that the amount of alcohol consumed has increased, whereas a negative increase indicates that alcohol consumption has decreased. The number of examinees who had almost no change in alcohol consumption $(\Delta 0)$ during the follow-up period was $16,003(64.8 \%)$. The number whose alcohol consumption increased was 4,309 , with $\Delta 1$ to $\Delta 4$ of $13.2 \%, 3.4 \%, 0.7 \%$, and $0.2 \%$, respectively. The number whose alcohol consumption decreased was 4,369 , with $\Delta-1$ to $\Delta-4$ of

Table 1 Changes in alcohol consumption during follow-up

\begin{tabular}{rrl}
\hline$\Delta$ & Number of cases & $(\%)$ \\
\hline+4 & 40 & $(0.2)$ \\
+3 & 181 & $(0.7)$ \\
+2 & 830 & $(3.4)$ \\
+1 & 3,258 & $(13.2)$ \\
0 & 16,003 & $(64.8)$ \\
-1 & 3,252 & $(13.2)$ \\
-2 & 805 & $(3.3)$ \\
-3 & 252 & $(1.0)$ \\
-4 & 60 & $(0.3)$ \\
\hline
\end{tabular}

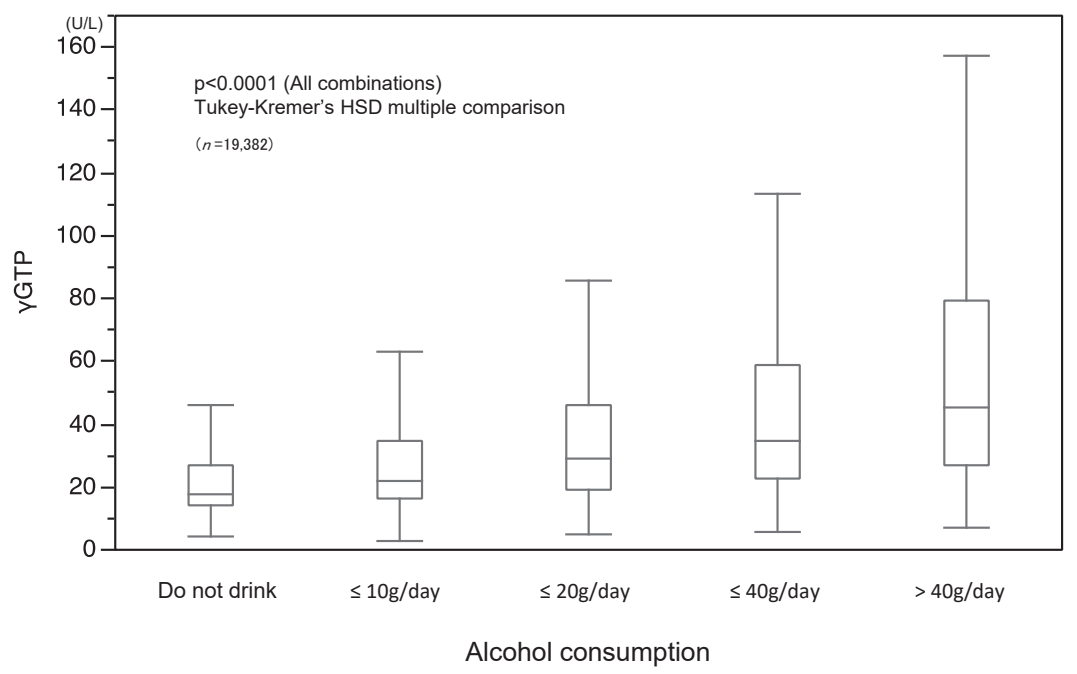

Fig. 1 The relationship between the alcohol consumption and $\gamma$-GTP, a marker of alcohol consumption, is shown. Alcohol consumption were divided into five groups: "Do not drink," " $\leq 10 \mathrm{~g} /$ day," " $\leq 20 \mathrm{~g} /$ day," " $\leq 40 \mathrm{~g} /$ day," and " $>40 \mathrm{~g} /$ day," and the value of $\gamma$-GTP was shown in a box plot. 
$13.2 \%, 3.3 \%, 1.0 \%$, and $0.3 \%$, respectively. The $\Delta 4$ and $\Delta-4$ groups were small, and in the subsequent analyses they were added together with the $\Delta 3$ and $\Delta^{-3}$ groups, respectively.

\section{Effects of change in alcohol consumption on lifestyle-related disease parameters}

With regard to obesity, BMI decreased when alcohol consumption decreased, and BMI increased when alcohol consumption increased $(p<0.0001)$. This relationship was nearly dose-dependent (Fig. 2 (a)). Blood pressure was assessed with SBP. With the exception of the $\geq \Delta 3$ group, SBP changed in parallel with changes in alcohol consumption $(\mathrm{p}<0.0001)$. In the $\leq \Delta-3$ group (nearly non-drinking group), the decrease in SBP was marked (Fig. 2 (b)). Next, looking at dyslipidemia, LDL-C did not change in the $\leq \Delta-3$ group, while a slight decrease was seen in the $\geq \Delta 3$ group. Increases were seen in all other groups $(p=0.0318$, Fig. 2 (c)). HDL-C changed nearly in parallel with changes in alcohol consumption $(p<0.0001$, Fig. 2 (d)). TG showed an evident decrease in the $\leq \Delta-3$ group and an evident increase in the $\geq \Delta 3$ group, but only slight changes in all other groups ( $p<0.0001$, Fig. 2 (e)). Diabetes was assessed with FBG, which improved nearly (a) Body mass index

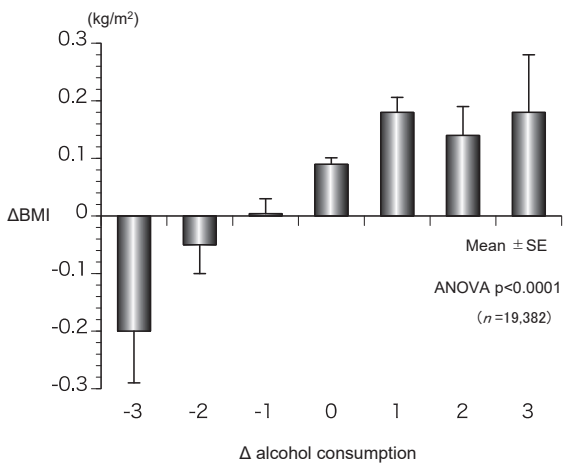

(d) HDL Cholesterol

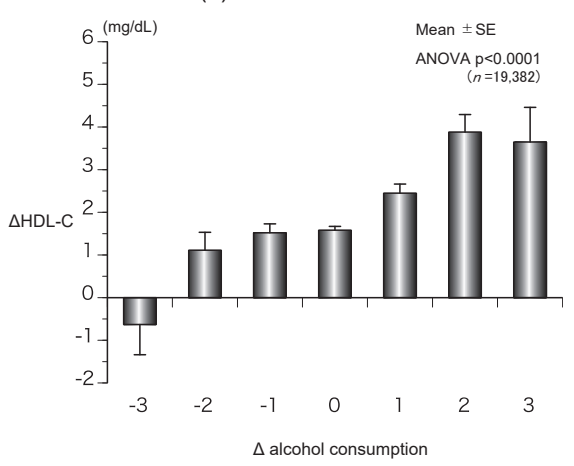

(b) Systolic blood pressure

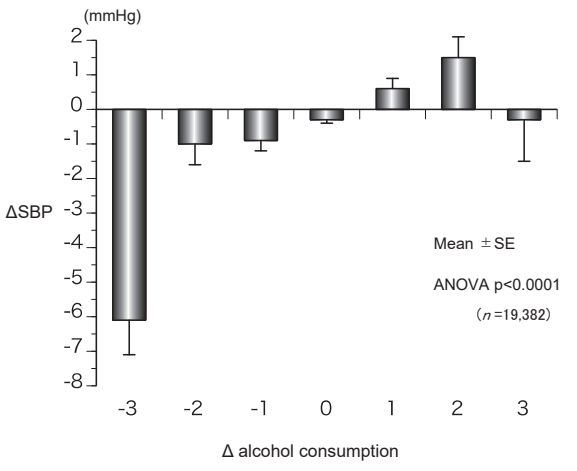

(e) Triglyceride

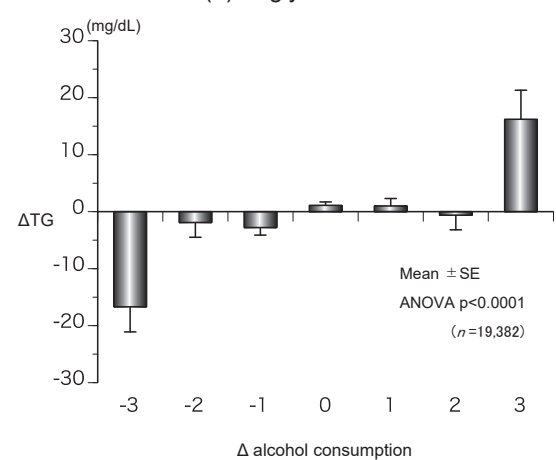

(g) Uric acid

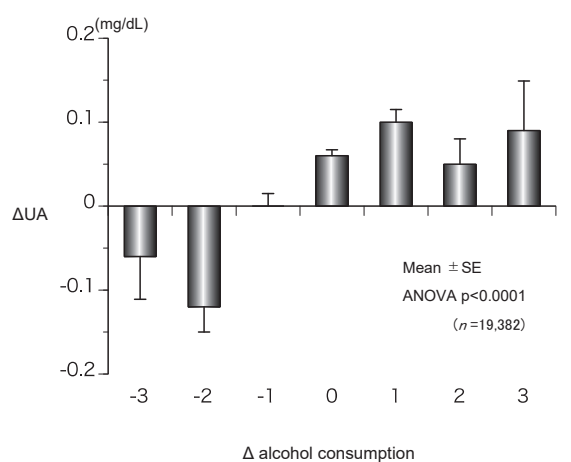

(c) LDL Cholesterol

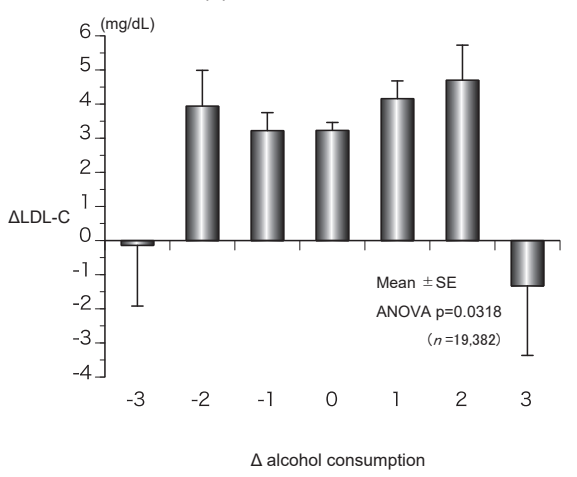

(f) Fasting blood glucose

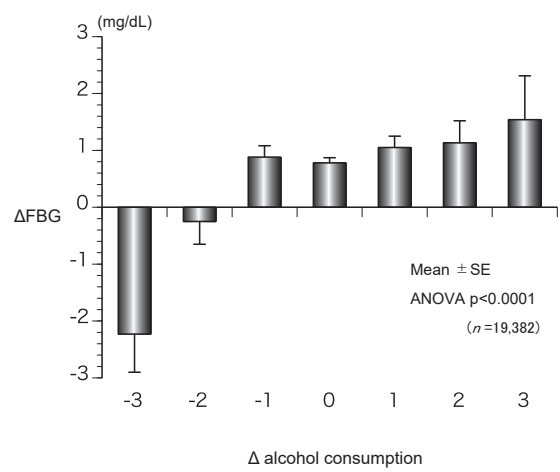

$\triangle$ alcohol consumption 
in parallel with decreases in alcohol consumption $(\mathrm{p}<0.0001$, Fig. 2 (f)). Changes in UA did not show consistent dose dependence with changes in alcohol consumption; however, UA levels increased in the group with increased alcohol consumption and decreased in the group with decreased alcohol consumption $(\mathrm{p}<0.0001$, Fig. 2 (g)).
4. Cases when factors in the development of fatty liver and metabolic syndrome worsened during the follow-up period

We investigated the effect on the development of fatty liver and metabolic syndrome (MetS) when the above-mentioned lifestyle-related disease parameters worsened during the follow-up period between check-ups at the Health Check-up Center. The

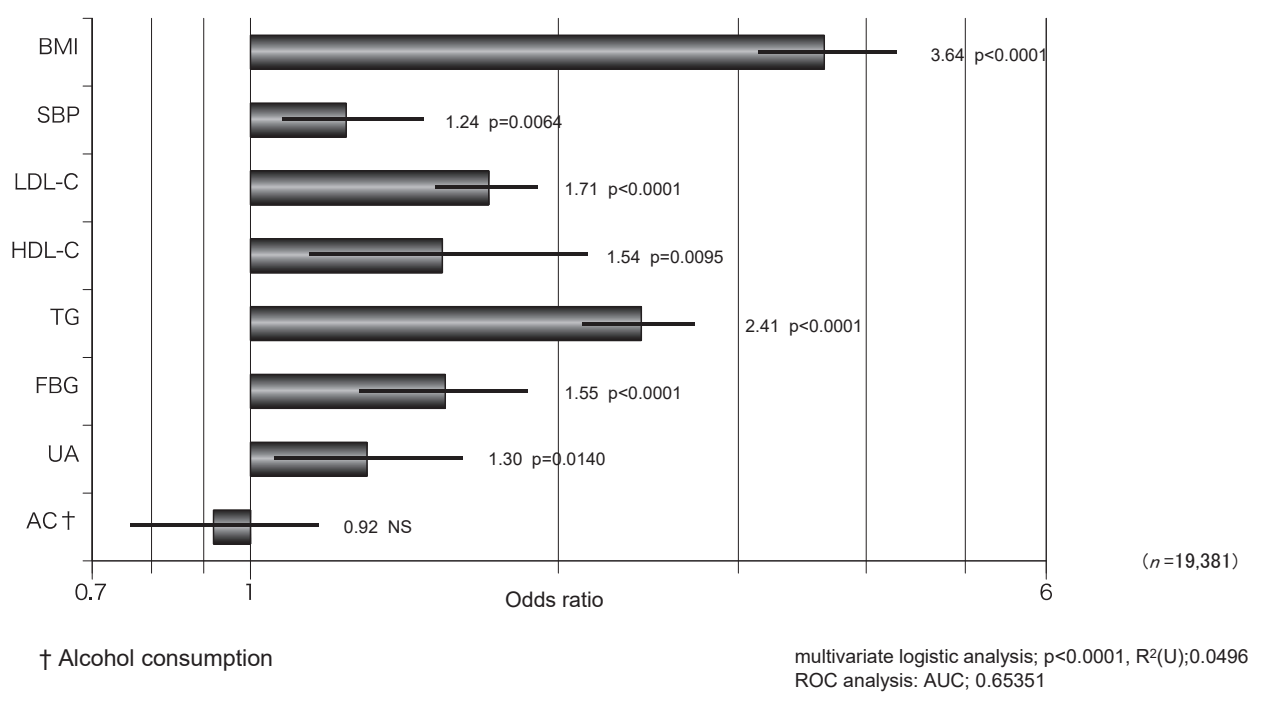

Fig. 3 With regard to the development of fatty liver, odds ratios were calculated for cases when BMI, SBP, LDL-C, HDL-C, TG, FBG, or UA exceeded their respective cut-off values, and when alcohol consumption exceeded $\leq 20 \mathrm{~g}$. BMI was found to be the most crucial, with an odds ratio of $3.64(p<0.0001)$, followed by TG with an odds ratio of $2.41(\mathrm{p}<0.0001)$. Below this, odds ratios were LDL-C 1.71, FBG 1.55, HDL-C 1.54, UA 1.30, and SBP 1.24, each of which was an independent exacerbation factor.

Table 2 Univariate analysis of the effect of alcohol consumption on the development of metabolic syndrome

\begin{tabular}{lccc}
\hline Alcohol consumption & Odds ratio & $95 \%$ confidence interval & p value \\
\hline More than $20 \mathrm{~g}$ /day & 1.613 & $1.022-2.433$ & 0.0404 \\
More than $40 \mathrm{~g}$ /day & 2.380 & $1.420-3.765$ & 0.0016 \\
\hline
\end{tabular}

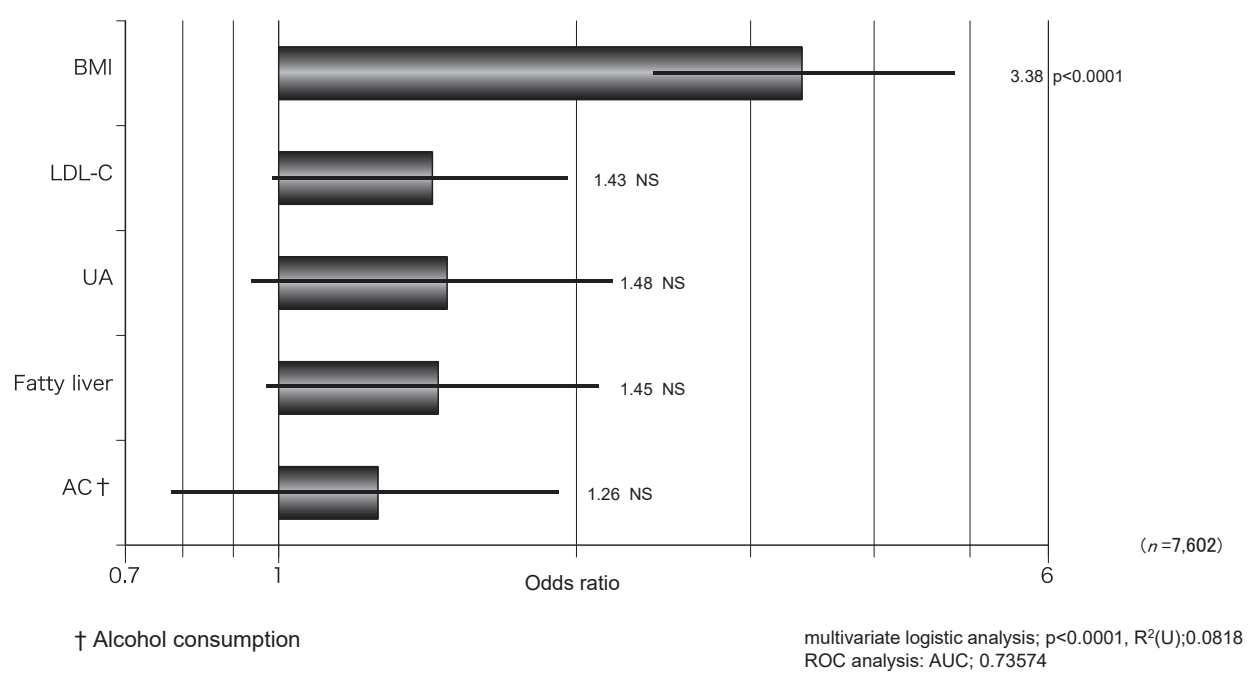

Fig. 4 MetS was investigated excluding SBP, HDL-C, TG, and FBG, which are included in the diagnostic criteria. The results showed that only worsening of BMI was significant, with an odds ratio of $3.38(\mathrm{p}<0.0001)$. 
analysis was done with a multivariate logistic analysis adjusted for age and sex. With regard to the development of fatty liver, odds ratios were calculated for cases when BMI, SBP, LDL-C, HDL-C, TG, FBG, or UA exceeded their respective cut-off values, and when alcohol consumption exceeded $\leq 20 \mathrm{~g}$. BMI was found to be the most crucial, with an odds ratio of 3.64 $(\mathrm{p}<0.0001)$, followed by $\mathrm{TG}$ with an odds ratio of 2.41 $(\mathrm{p}<0.0001)$. Below this, odds ratios were LDL-C 1.71, FBG 1.55, HDL-C 1.54, UA 1.30, and SBP 1.24, each of which was an independent exacerbation factor (Fig. 3). In this study, however, increased alcohol consumption was not an independent factor for the development of fatty liver.

The MetS development by the alcohol consumption was analyzed by the univariate analysis. The Odds ratios for the development of MetS were 1.613 for alcohol consumption more than 20 $\mathrm{g}$ /day and 2.38 for alcohol consumption more than $40 \mathrm{~g}$ /day (Table 2). Next, MetS was investigated excluding SBP, HDL-C, $\mathrm{TG}$, and FBG, which are included in the diagnostic criteria. The results showed that only worsening of BMI was significant, with an odds ratio of $3.38(\mathrm{p}<0.0001)$ (Fig. 4). Worsening of LDL-C and UA and development of fatty liver tended to contribute to the development of MetS, but they were not significant.

\section{Discussion}

This study examined how various lifestyle-related disease parameters changed when alcohol consumption changed. Since the accurate estimation of the alcohol consumption is self-report by the questionnaire method, it is quite difficult. In addition, there was a problem in the quantification, because the alternatives of the questionnaire used in our hospital's Health Check-up Center were "do not drink," "1 day a week," "2 to 5 days a week," and "6 or more days per week." Especially, the handling of " 2 to 5 days" was difficult. Since the number of medical examinees who choose it decreases as the alcohol consumption increases, the possibility that there are more medical examinees who drink "2-3 days" than "4-5 days" within a week was considered. The simple average is 3.5 days/week $(=1 / 2)$, but the coefficient is set at $1 / 3$ considering the effect of the weighting. The alcohol consumption was estimated on the basis of this, and a good dose-dependent relationship was found between the alcohol consumption calculated as shown in Fig. 1 and $\gamma$-GTP, a marker of drinking. In addition, $\gamma$-GTP is increased in fatty liver and obesity, and on the other hand, there is a group (non-responder) which does not increase in spite of drinking, however, the effect is not large, because there is sufficient $n$ number in this result.

The people who undergo health check-ups are a very heterogeneous group and it is highly unlikely that drinking habits alone change during the follow-up period. Various background factors must be viewed to change simultaneously. Thus, there should be very great variability in the observed values. Despite this, the very large number of subjects should give validity to the mean values (shown with standard error to estimate the population mean). Even when the amount of change is small, there is thought to be a causal relationship when a dose-dependent relationship exists between the change in alcohol consumption and the changes in the observed values. In this study, all factors except for LDL-C were dose-dependent on changes in alcohol consumption. It is difficult to evaluate repeaters that have been seen more than three times. This is because only the first and last data are taken into consideration, and the information is not reflected, for example, when the amount of drinking largely changes and returns to the original level in the long term. This is considered to be a future issue.

First, BMI was investigated as an indicator of obesity. In general, alcohol consumption is thought to promote obesity, and in fact a positive correlation between consumption of a large amount of alcohol and obesity has been reported ${ }^{3)}$. In contrast, in a previous report, we showed the possibility that small amounts of alcohol of $\leq 20$ g significantly decrease the frequency of obesity ${ }^{2)}$. The present result that BMI decreased when alcohol consumption decreased and increased when alcohol consumption increased does not contradict those findings. With regard to hypertension, an investigation of people who undergo health check-ups showed a relationship between increased alcohol consumption and blood pressure, a tendency that is considered to be stronger in elderly people ${ }^{4}$. In a previous report, we found that the risk of hypertension rose in a dose-dependent manner with alcohol consumption of $\geq 40 \mathrm{~g}$, but we did not see a significant difference with small amounts ${ }^{2}$. This is related to the finding in this study of little change in the groups from $\Delta-2$ to $\Delta 2$. The reason for little change in blood pressure above $\Delta 3$ is unclear. A recent meta-analysis showed that in men, high alcohol consumption was associated with risk of hypertension while in women alcohol consumption and risk of hypertension showed a J-shaped curve, indicating a sex difference ${ }^{5)}$. For dyslipidemia, we investigated the three parameters of LDL-C, HDL-C, and TG. In our previous report, we showed that the frequency of hyper-LDL cholesterolemia decreased in a group with low alcohol consumption ${ }^{2}$. Similar conclusions have been reported for $\operatorname{men}^{6)}$ and women ${ }^{7}$. However, in our present investigation of changes in alcohol consumption, a consistent dose-dependent trend was not seen and it was difficult to interpret the findings; there was no change in the group with a large decrease in alcohol consumption and a slight decrease in the group with a large increase, but the other groups showed an increase in LDL-C. For HDL-C, in contrast, changes in alcohol consumption and changes in HDL-C moved together in a dosedependent manner. This is in agreement with the many reports showing a positive correlation in which HDL-C is strongly dependent on amount of alcohol consumed ${ }^{2,4,6-8)}$. TG greatly decreased in the $\leq \Delta-3$ group, and greatly increased in the $\geq \Delta 3$ group. The change in alcohol consumption was a negative factor for TG in the group with low alcohol consumption but was a positive factor in the moderate to high consumption groups. Our previous report showed that the border was around $20 \mathrm{~g}^{2}$. This is thought to be the likely reason for the small amount of change in the $\Delta-2$ to $\Delta 2$ groups. It has been reported that a positive correlation is seen between alcohol consumption and TG levels in men ${ }^{6}$, whereas it has been reported that no significant difference is seen in women ${ }^{7}$. With respect to diabetes, an examination of FBG showed that it improved nearly in parallel with decreases in alco- 
hol consumption. This agrees with our previous report that alcohol consumption of $\geq 20 \mathrm{~g}$ is a positive factor for elevated FBG, and that the odds ratio was dose-dependent $t^{2}$. Many previous reports, however, have suggested that small amounts of alcohol have a protective effect against type 2 diabetes. This has also been demonstrated in meta-analyses ${ }^{9,10)}$. From an investigation of insulin resistance, it was also reported that alcohol is an independent negative factor for homeostasis model assessment of insulin resistance ${ }^{11)}$. Although a consistent dose dependence was not shown for UA, it was found that UA levels increased in the groups with increased alcohol consumption and decreased in the groups with decreased alcohol consumption. This supports past reports $^{2,12,13)}$.

Next, we investigated the effects when factors in the development of fatty liver worsened during the follow-up period. With respect to factors affecting the development of fatty liver, in a past study in which we examined 21,493 subjects $^{14)}$ the odds ratio with BMI of $\geq 25 \mathrm{~kg} / \mathrm{m}^{2}$ was 5.81 , followed by 3.04 with TG of $\geq 150 \mathrm{mg} / \mathrm{dL}$. Beyond that, the odds ratios were 2.01 with male sex, 1.99 with $F B G \geq 110 \mathrm{mg} / \mathrm{dL}, 1.82$ with HDL-C $\leq 39 \mathrm{mg} / \mathrm{dL}$, 1.66 with LDL-C $\geq 140 \mathrm{mg} / \mathrm{dL}, 1.55$ with $\mathrm{UA} \geq 7.1 \mathrm{~mL}, 1.46$ with SBP $\geq 130 \mathrm{mmHg}, 1.22$ with age of $\geq 50$ years old, and 0.74 with alcohol consumption of $\leq 20 \mathrm{~g}$ /day (all $\mathrm{p}<0.0001$ ). Small amounts of alcohol have a protective action against the development of fatty liver, but the odds ratios were $0.77(p<0.0001)$ with consumption of $40 \mathrm{~g} / \mathrm{day}, 0.87$ (not significant) with $60 \mathrm{~g}$, and 1.18 (not significant) with $80 \mathrm{~g}$. Although significant differences were not seen, the protective effect disappeared together with increases in alcohol consumption ${ }^{14}$. The order of these odds ratios was somewhat different compared with the odds ratios with worsened factors in this study, but the trends were generally the same. Increased alcohol consumption (more than $20 \mathrm{~g}$ ) was not an independent factor for the development of fatty liver, but it also did not contribute protectively.

MetS was investigated with the exclusion of SBP, HDL-C, TG, and $\mathrm{FBG}$, which are included in the diagnostic criteria. As a result, only worsening of BMI was significant, with an odds ratio of $3.38(p<0.0001)$. Worsening of LDL-C and UA and the development of fatty liver tended to contribute to the development of MetS, but they were not significant. Univariate analysis showed a correlation between increased alcohol consumption and MetS incidence. In the meantime, in the multivariate analysis, the increase in the alcohol consumption seemed not to be an independent factor of the MetS crisis, and it was indirect through the aggravation of SBP, TG, FBG and BMI included in diagnostic criteria of MetS. Therefore, drinking to a degree that does not cause deterioration of parameters of lifestyle-related diseases may be acceptable from this viewpoint. High alcohol consumption clearly leads to alcoholic fatty liver. In light to moderate drinkers, the relationship with MetS factors other than hypertension is reported to be lower than that of non-drinkers ${ }^{4}$. In contrast, high alcohol consumption is reported to be associated with an increased prevalence of MetS ${ }^{15)}$.

Alcohol consumption is a lifestyle habit and a preference. It is also an aspect of culture. At the same time, it is a cause of various diseases that produce chronic organ damage. It is also a cause of various accidents resulting from alterations in consciousness, and damages not only physical but also mental health due to dependency. It thus has a fair number of social disadvantages. Drinking behavior does not exist in isolation, but is involved in complex ways with many factors, including excessive calorie intake, salt intake, nutritional imbalance, smoking, stress, and sleep. Because of this, the effects of alcohol and its relationship with various diseases are quite diverse, depending on the report. Among such reports, the relationship between alcohol and cardiovascular death or all-cause mortality has been well studied, and is known to show a $\mathrm{J}$ curve ${ }^{16,17)}$. It is generally explained that small amounts of alcohol reduce the risk of ischemic heart disease by improving lipid and lipoprotein risk factors, whereas large amounts of alcohol increase the risk of cerebral hemorrhage and hypertensive disorders via elevated blood pressure ${ }^{7}$. There are also many reports showing $\mathrm{J}$ curves between alcohol and overall mortality in prospective cohort studies with Japanese subjects ${ }^{18-21)}$. The risk of cancer $^{22)}$ and hypertension ${ }^{23,24)}$ has also been shown to increase linearly together with alcohol consumption. Thus, while there are many reports indicating the health benefits of small amounts of alcohol, we should not recommend alcohol to non-drinkers ${ }^{25)}$. The reasons are as follows: Nearly all of these studies, including this one, are cross-sectional studies and look only at the relationship between alcohol and various factors. While there are prospective, large-scale cohort studies of mortality, very few studies have shown any kind of benefit in randomized trials with nondrinking subjects and thus the possibility of detriments from drinking cannot be ruled out even in individuals thought to be at low risk of disease ${ }^{25)}$. A recent large-scale meta-analysis ${ }^{26)}$ reported that the amount of alcohol to minimize the overall health loss risk is zero drinks per day, which has attracted much attention. Today, it is important to spread information to the effect that pure alcohol of approximately $20 \mathrm{~g}$ per day on average (a smaller amount for women) is "temperate, moderate alcohol intake" as recommended by the Japanese Ministry of Health, Labour and Welfare.

The authors state that they have no Conflict of Interest (COI).

\section{REFERENCES}

1) Roerecke M, Rehm J. Alcohol intake revisited: risks and benefits. Curr Atheroscler Rep 2012; 14: 556-62.

2) Kojima S, Ito $H$, Takashimizu S, Mizukami H, Nagata J, Ichikawa $\mathrm{H}$, et al. The influence of drinking based on data from health check-up. Jpn J Alcohol \& drug dependence 2015; 50: 14457.

3) Shelton NJ, Knott CS. Association between alcohol calorie intake and overweight and obesity in English adults. Am J Public Health 2014; 104: 629-31.

4) Wakabayashi I. Influence of age on the relationship between alcohol consumption and metabolic syndrome. Gerontology 2012; 58: 24-31.

5) Briasoulis A, Agarwai V, Messerli FH. Alcohol consumption and the risk of hypertension in men and women: a systematic review and meta-analysis. J Clin Hypertens 2012; 14(11): 792-8. 
6) Nakanishi N, Tatara K, Nakamura K, Suzuki K. Association of lifestyle with serum lipid levels: a study of middle-aged Japanese men. J Epidemiol 2000; 10(4): 216-25.

7) Nanchahal K, Ashton WD, Wood DA. Alcohol consumption, metabolic cardiovascular risk factors and hypertension in women. Int J Epidemiol 2000; 29: 57-64.

8) Ellison RC, Zhang Y, Qureshi MM, Knox S, Arnett DK, Province MA. Lifestyle determinants of high-density lipoprotein cholesterol: the national heart, lung and blood institute family heart study. Am Heart J 2004; 147: 529-35.

9) Koppes LL, Dekker JM, Hendriks HF, Bouter LM, Heine RJ. Moderate alcohol consumption lowers the risk of type 2 diabetes: a meta-analysis of prospective observational studies. Diabetes Care 2005; 28(3): 719-25.

10) Baliunas DO, Taylor BJ, Irving H, Roerecke M, Patra J, Mohapatra $\mathrm{S}$, et al. Alcohol as a risk factor for type 2 diabetes; a systematic review and meta-analysis. Diabetes Care 2009; 32(11): 2123-32.

11) Fueki $Y$, Miida $T$, Wardaningsih $E$, Ito $M$, Nakamura $A$, Takahashi A. et al. Regular alcohol consumption improves insulin resistance in healthy Japanese men independent of obesity. Clin Chim Acta 2007; 382(1-2): 71-6.

12) Nakanishi N, Tatara $K$, Nakamura $K$, Suzuki K. Risk factors for the incidence of hyperuricemia: a 6-year longitudinal study of middle-aged Japanese men. Int J Epidemiol 1999; 28(5): 888-93.

13) Shiraishi $H$, Une $H$. The effect of the interaction between obesity and drinking on hyperuricemia in Japanese male office workers. J Epidemiol 2009; 19(1): 12-6.

14) Kojima $S$, Ito $H$, Takashimizu $S$, Shirai $T$, Ichikawa $H$, Nagata J, et al. A small amount of alcohol drinking may suppress the development of fatty liver (in Japanese). Alcohol and Biomedical Research 2016; 34: 76-80.

15) Nakashita $Y$, Nakamura M, Kitamura A, Kiyama M, Ishikawa $Y$, Mikami H. Relayionships of cigarette smorking and alcohol consumption to metabolic syndrome in Japanese men. J Epidemiol 2010; 20(5): 391-7.

16) Costanzo S, Di Castelnuovo A, Donati MB, Iacoviello L, de Gaetano, G. Alcohol consumption and mortality in patients with cardiovascular disease: a meta-analysis. J Am Coll Cardiol 2010; 55(13): 1339-47.
17) Xi B, Veeranki SP, Zhao M, Ma C, Yan Y, Mi J. Relationship of alcohol consumption to all-cause, cardiovascular, and cancerrelated mortality in U.S. adults. J Am Coll Cardiol 2017; 70(8): 913-22.

18) Lin $Y$, Kikuchi S, Tamakoshi A, Wakai K, Kawamura T, Iso H, et al. Alcohol consumption and mortality among middle-aged and elderly Japanese men and women. Ann Epidemiol 2005; 15(8): 590-975.

19) Sadakane A, Nakamura Y, Gotoh T, Ishikawa S, Kayaba K. Amount and frequency of alcohol consumption and all-cause mortality in a Japanese population: the JMS cohort study. J Epidemiol 2009; 19(3): 107-15.

20) Inoue $M$, Nagata $C$, Tsuji I, Sugawara $Y$, Wakai K, Tamakoshi A, et al. Impact of alcohol intake on total mortality and mortality from major cause in Japan: a pooled analysis of six large-scale cohort studies. J Epidemiol Community Health 2012; 66(5): 44856.

21) Saito E, Inoue M, Sawada N, Chavat H, Shimazu T, Yamaji T, et al. Impact of alcohol intake and drinking patterns on mortality from all causes and major causes of death in a Japanese population. J Epidemiol 2018; 283: 140-8.

22) Inoue $M$, Tsugane $S$. Impact of alcohol drinking on total cancer risk: data from a large-scale population-based cohort study in Japan. Br J Cancer 2005; 92(1): 182-7.

23) Nakanishi N, Makino K, Nishina K, Suzuki K, Tatara K. Relationship of light to moderate alcohol consumption and risk of hypertension in Japanese male office workers. Alcohol Clin Exp Res 2002; 26(7): 988-94

24) Ohmori S, Kiyohara $Y$, Kato I, Kubo M, Tanizaki $Y$, Iwamoto H, et al. Alcohol intake and future incidence of hypertension in a general Japanese population: the Hisayama study. Alcohol Clin Exp Res 2002; 26(7): 1010-6.

25) O’Keefe JH, Bhatti SK, Bajwa A, DiNicolantonio JJ, Lavie CJ. Alcohol and cardiovascular health: the dose makes the poison...or the remedy. Mayo Clin Proc 2014; 89(3): 382-93.

26) Griswold MG. Alcohol use and burden for 195 countries and territories, 1990-2016: a systematic analysis for the Global Burden of Disease Study 2016. Lancet 2018; 392(10152): 1015-35. 